\title{
A familial pericentric inversion of chromosome 22 with a recombinant subject illustrating a 'pure' partial monosomy syndrome
}

\author{
J L WATT, I A OLSON, A W JOHNSTON, H S ROSS, \\ D A COUZIN, AND G S STEPHEN
}

From the Department of Genetics, University of Aberdeen, Foresterhill, Aberdeen AB9 2ZD.

SUMmARY A family in which a pericentric inversion of chromosome 22 , inv(22)(p11q12), is segregating is described. Special reference is made to a unique recombinant subject with a 'pure' partial monosomy 22 syndrome of maternal origin. An attempt has been made to correlate the phenotypic abnormalities with monosomy for the segment $22 q 12 \rightarrow$ qter.

Pericentric inversions are two break events which are much less frequent than translocations (incidence $0.01 \%) .{ }^{1}$ In addition to the relatively common pericentric inversion of chromosome $9,{ }^{2}$ now considered to be a harmless polymorphism, pericentric inversions have been described for most human chromosomes. We are aware of only two other reports of pericentric inversion of chromosome $22 .^{34}$

We describe here a family in which a pericentric inversion of chromosome 22 is segregating, including what we believe to be the first case of 'pure'

Received for publication 29 July 1984.

Accepted for publication 29 August 1984.

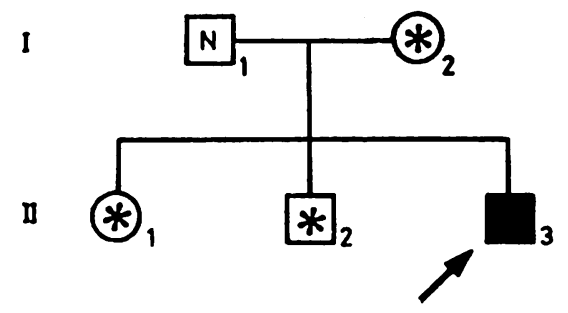
(N) N Normal chromosomes

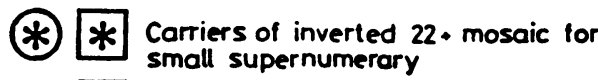
Rec (22), dup p, inv (22)(pllq21)

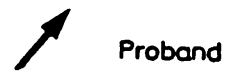

FIG 1 Family pedigree. partial monosomy 22 syndrome resulting from maternal meiotic recombination. The familial situation is made more complex by the fact that there is a small marker chromosome segregating along with the balanced inversion in three family members (fig 1).

\section{Case reports}

The proband (II.3, fig 1) was born on 14.3.69. At the age of 14 his height was $143.5 \mathrm{~cm}$ and he had microcephaly (head circumference $53 \mathrm{~cm}$ ). His IQ remains too low to test adequately and he has virtually no speech. His face is rounded with full cheeks and eyebrows, marked epicanthic folds, and

TABLE Main features of ring 22 syndrome compared to the pure monosomy 22 in the proband.

\begin{tabular}{|c|c|c|}
\hline & $\begin{array}{l}\text { After } \\
\text { Funderburk et at }\end{array}$ & Proband \\
\hline IQ & $50^{*}$ & Too low to test \\
\hline Height & 3rd centile* & $143.5 \mathrm{~cm}$ \\
\hline Head circumference & 3rd centile & $53 \mathrm{~cm}$ \\
\hline Epicanthus & $+^{*}$ & + \\
\hline Eyes & & Deep set \\
\hline Eyebrowş & Full* & Full and long \\
\hline Eyelids & & Full \\
\hline Ears & Large, abnormal* & Normal \\
\hline Cheeks & Flat & Full \\
\hline Lips & Thick, full* & Normal, slightly long philtrum \\
\hline Dental malocclusion & + & Slight \\
\hline Spine & Lordosis/kyphosis* & Lordosis \\
\hline Fingers & Syndactyly & Short with clinodactyly \\
\hline Toes & Syndactyly & Syndactyly \\
\hline Muscle tone & Hypotonic* & Normal \\
\hline Gait & Ataxic* & Awkward \\
\hline Fits/abnormal EEG & Present & None \\
\hline
\end{tabular}

*Commonest findings. 
deep set eyes. The hands and fingers are short with incurved little fingers. His trunk is rather long and his gait is awkward and flat footed. Further details are given in the table.
Cases I.2 and II.1 (fig 1) resemble each other and although case II.2 shows some similarities he is of $\stackrel{?}{+}$ normal stature. All three are microcephalic and of $\bar{c}$ borderline intelligence. Their eyebrows are long,
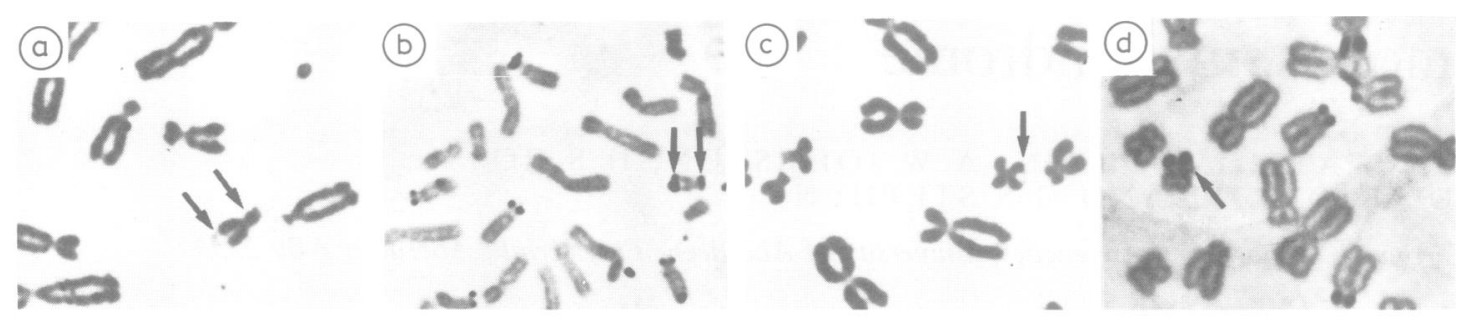

(e)
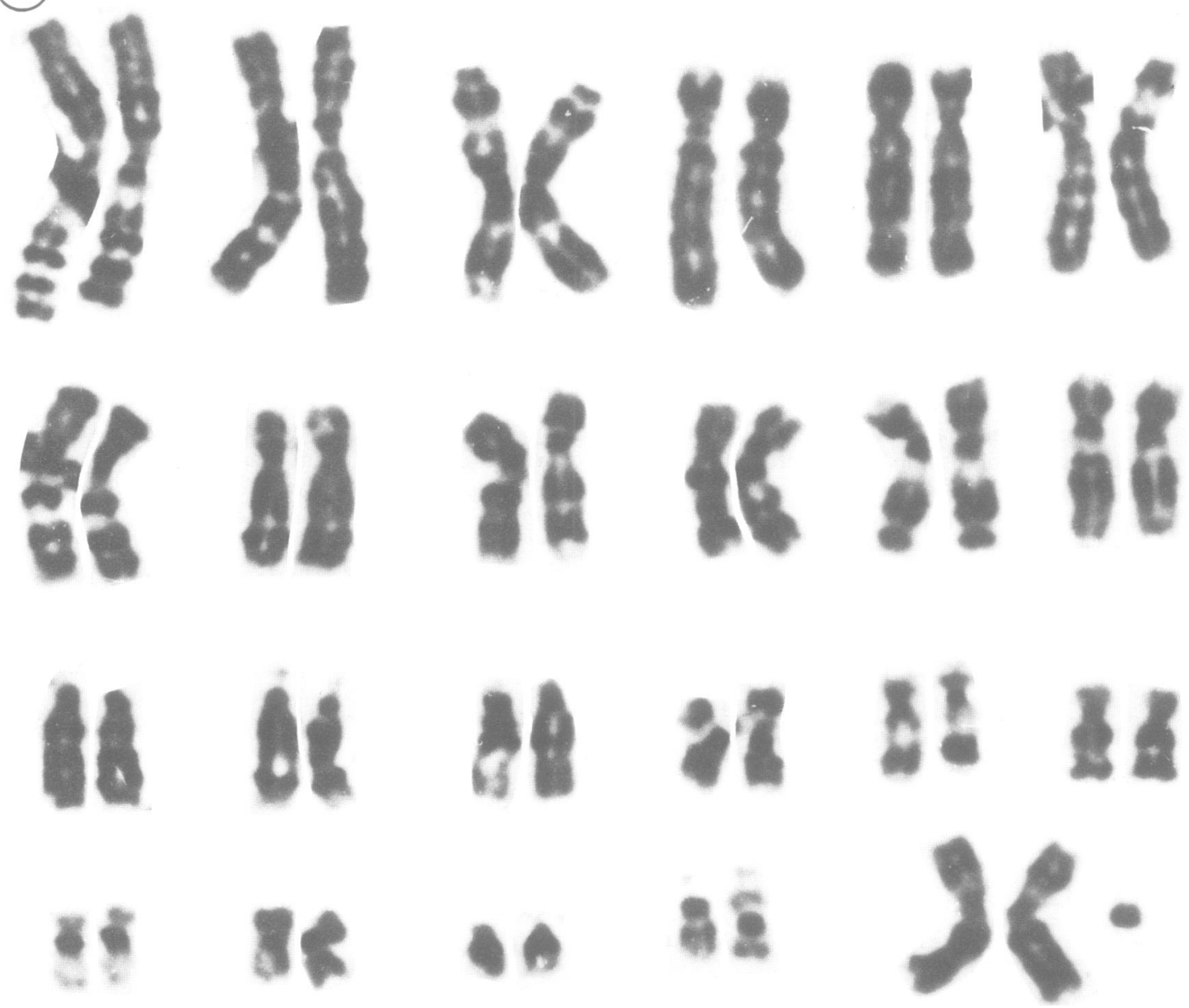

FIG 2 ( $a$ and $b$ ) Partial metaphases from the proband showing bisatellite 22 by orcein staining and Ag-NOR staining.

( $c$ and d) Partial metaphases showing chromosome 22 with pericentric inversion by orcein staining and Ag-NOR staining. (e) $G$ banded karyotype from 1.2 showing inverted 22 and small marker (? ring 22). 
tending to curve downwards at the outer ends. Cases I. 2 and II. 2 also have asymmetry of the skull and face, giving II. 2 in particular an oriental look ('doe's eyes').

\section{Materials and methods}

Chromosome analysis was performed on peripheral blood cultures. The resulting preparations were treated with $\mathrm{G}$ banding, $\mathrm{C}$ banding, DA/DAPI fluorescence, and Ag-NOR staining.

\section{Results}

The proband was found to have 46 chromosomes including a marker chromosome 22 with satellites at both ends (fig $2 \mathrm{a}$ ). This marker chromosome has an active nucleolar organiser at each end, as shown by positive Ag-NOR staining (fig 2b), and contains no clear component of chromosome 15 (DA/DAPI negative). The findings in the proband were confirmed independently in Glasgow. The father has an apparently normal $46, X Y$ male karyotype, while the mother (I.2), brother (II.2), and sister (II.1) all carry a pericentric inversion of chromosome 22: inv(22)(p11q12) (fig 2c, d). In addition to this chromosome abnormality, these three subjects are mosaic for an additional small marker chromosome (fig 2e). The origin of this tiny supernumerary could not be identified by any of the banding methods used. The proportion of cells carrying the supernumerary varies, with the mother and brother having $16 \%$ and the sister having $75 \%$. Sixty cells from the index case were analysed thus excluding $5 \%$ mosaicism with $95 \%$ confidence. ${ }^{6}$

The proband (II.3) can therefore be considered to carry a recombinant of the familial pericentric inversion. The three family members with the balanced inversion (I.2, II.1, and II.2) are also mosaic for a small supernumerary chromosome and can therefore be thought of as having partial mosaic trisomy.

\section{Discussion}

It is generally accepted that pericentric inversions are balanced rearrangements and as such should not affect the phenotype of the carrier. It was once thought that pericentric inversions were also innocuous with reference to fertility and offspring. ${ }^{7}$ Generally speaking, (heterozygous) small inversions are unlikely to cause reproductive problems since the short inverted regions usually remain unpaired at meiosis, and even in the event of pairing, a crossover is rarely formed in the critical loop area (fig 3).

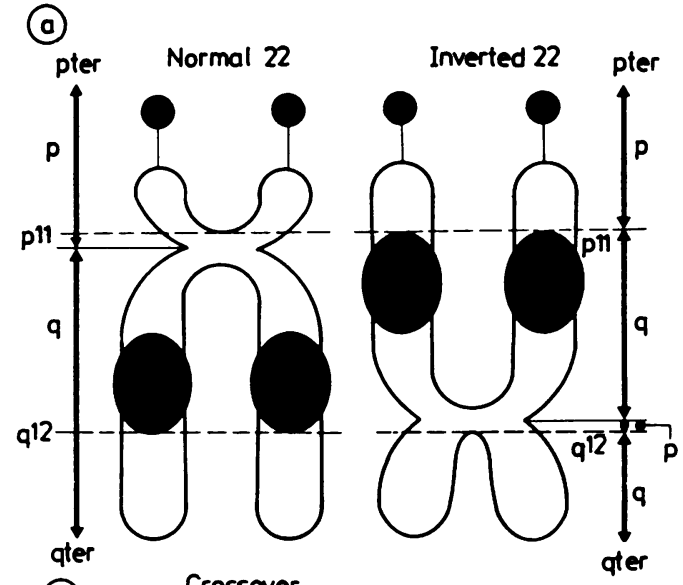

(b)
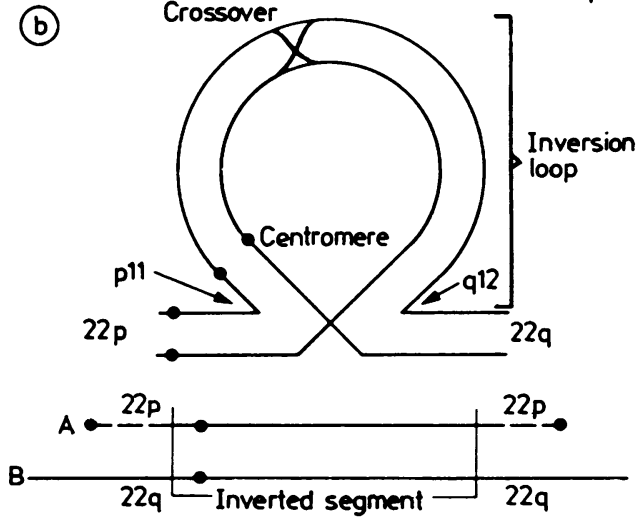

$A$ and B. Two types of recombinant chromosomes are possible. The proband in the present family has type A

(c)

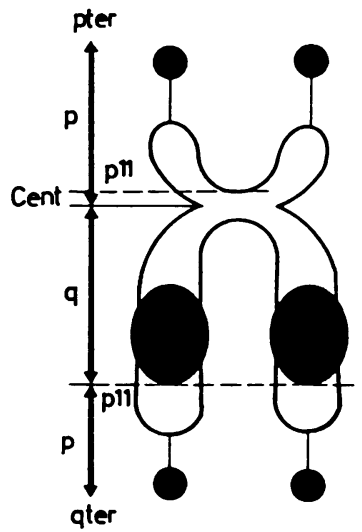

Recombinant 22 (type A)

(Monosomic for 22q 12 $\rightarrow$ qter)

FIG 3 (a) Diagram of normal and inverted chromosome 22 with breakpoints. (b) Diagram to illustrate likely meiotic events leading to two types of recombinant chromosome ( $A$ and $B$ ). (c) Diagram of recombinant (type A) seen in proband. 
As a sequel to the observation that trisomy is more common than monosomy in man, the type of recombinant offspring with the larger duplication and smaller deletion is considered more viable in the case of pericentric inversions. This is demonstrated in both cases of inverted chromosome 22 reported by Cantu et $a l^{3}$ and Fujimoto et al. ${ }^{4}$ Our case is exceptional in that we describe the rarer alternative in the proband, representing a large monosomy 22 (q12 $\rightarrow$ qter).

It would be most satisfactory if the two phenomena of the inversion (with its recombinant) and the unidentified supernumerary could be mechanistically related. This is possible if it is assumed that the supernumerary is in fact a ring chromosome consisting only of the inverted region of 22, that is, $\mathrm{r}(22)(\mathrm{p} 1 \mathrm{lq} 12)$. The diameter of such a ring would be about one-third the length of the inverted segment, which is indeed the case. A ring, along with an acentric fragment, is the asymmetrical equivalent of an inversion. Which one forms depends simply on the chance aberrant rejoining at the breakpoints on each arm of a chromosome in forming an inter-arm intrachange.

It is postulated that both types of aberrant rejoining may occur simultaneously, one in each of the two chromatids in a dividing cell during, or perhaps even after, $S$ phase (fig 4). If, in addition, at the subsequent anaphase the inverted and ring chromatids fail to separate then there will result a cell with both a ring and an inverted chromosome. The fragment will, of course, subsequently be lost. The tendency of the ring to non-disjunction would explain its mosaicism in the inversion carriers.

Recombination involving a pericentric inversion of an acrocentric chromosome yields pure monosomy or trisomy in recombinant subjects. It is not complicated by the presence of short arm imbalance, because they are relatively inert genetically containing only rRNA genes. We are therefore able to describe a "pure' partial monosomy 22 syndrome, since the trisomy for $22 p$ is believed to have a negligible effect on the phenotype. This reasoning has been used to describe pure partial trisomy of chromosomes $14^{8}$ and $22 .{ }^{3}$ The proband (II.3) is therefore monosomic for the distal half of $22 \mathrm{q}$ $(q 12 \rightarrow q$ ter $)$. The clinical signs in the relatives of the proband are probably the consequence of trisomy for the supernumerary modified by the mosaicism, rather than the inversion per se. On the other hand. the marked mental retardation of the proband together with the other phenotypic abnormalities is considered to be due to the substantial monosomy of chromosome 22, since his chromosome anomaly is the direct result of maternal meiotic recombination and he does not carry the supernumerary. (a) Mitotic loop before breakage

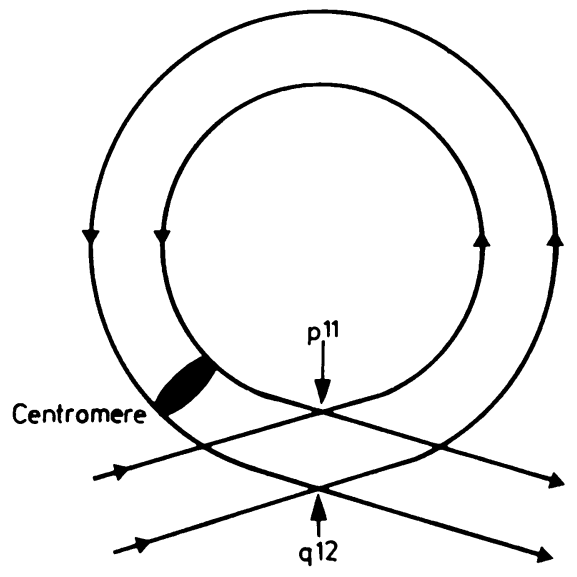

(b) The alternative ways of rejoining
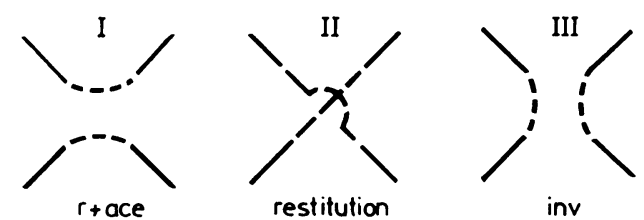

inv

(c) Mitotic loop after rejoining

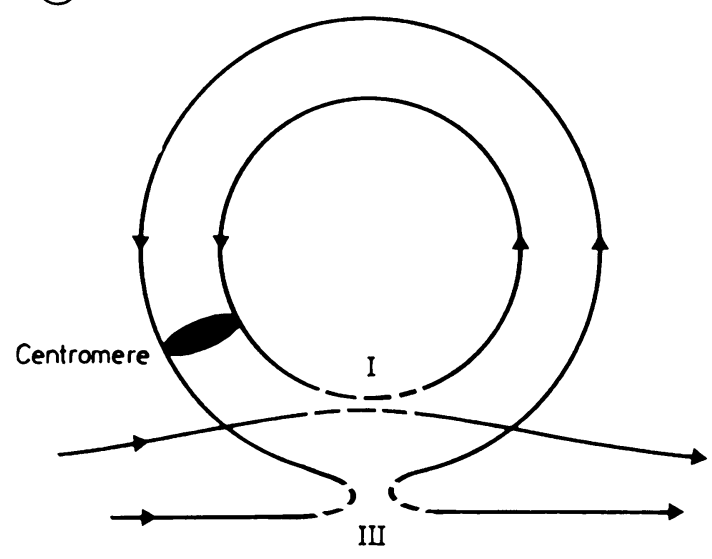

Fig 4 Diagrams illustrating the possible common origin of the inversion and the small ring. These events are postulated to occur during late'S phase. (a) Double stranded chromosome 22 looped round so that breakpoints are in juxtaposition. (b) Threc alternative ways of rejoining following breakage of all four strands. (c) Alternative's I and $\stackrel{\mathbb{Q}}{\mathrm{Q}}$ III are thought to have occurred simultaneously. 
The nearest equivalent deletion to this recombinant is in persons with $46, \mathrm{r}(22)$, noted to have variable clinical features," 10 due to the mosaicism which typically occurs with ring instability. Hunter $e t$ $a l^{11}$ specifically refer to some minor signs, including long eyebrows and short hands, which were present in our patient, but also to mild maxillary hypoplasia, which was not. The full eyebrows mentioned in several reports seem also to be associated with fullness of the upper eyelid, as in our patient.

Funderburk et al,$^{5}$ following Hunter et al.$^{11}$ have tabulated the principal findings in the reported cases of ring 22. Although there are certain features in common (table), there are also notable differences, for example, in facial features. The first patient of Funderburk et al, ${ }^{5}$ like ours, as well as case 2 of Hunter et al, ${ }^{11}$ also has a bulbous nose.

Apart from mental and physical retardation, the suggestive features of partial monosomy for chromosome 22 are epicanthic folds, full eyebrows and upper eyelids, deep set eyes, kyphosis or lordosis, and short fingers. ${ }^{12}$ Hypotonia, ataxia, and epilepsy have also been reported, while in our patient a broad bulbous nose, long eyelashes, and long philtrum were notable findings.

The family members illustrating the presumptive partial mosaic trisomy for $22(\mathrm{p} 11 \rightarrow \mathrm{q} 12)$ show several features in common, as detailed in the introduction. Various attempts have been made to correlate the physical signs in trisomy 22 with specific sites, but the resulting suggestions have conflicted. ${ }^{3}{ }^{1.3}$ This is partly because some signs are non-specific, partly because of mosaicism, and partly because of variation in the amount of extra chromosome 22 material.

Mental and physical retardation, microcephaly, cryptorchidism, congenital heart disease, cleft palate, a long beaked nose, and long philtrum appear to be the common features. Of these only microcephaly was present in all three of our patients, though two also had craniofacial asymmetry. Two of the three (I.1 and II.2) are probably retarded and I.1 and II.1 are only 147 and $151 \mathrm{~cm}$ tall. The probable explanation of the phenotypic variation is the presence of mosaicism.

In view of our unifying hypothesis for explaining the abnormalities in this family (fig 4), it is suggested that mosaicism for rings may be usefully looked for in families segregating for small pericentric inversions. This is especially so when there are clinical signs in non-recombinant persons after initial analysis. With segregation of the progenitor inversion and ring the possibility also exists that these aberrations may be found separately in different members of a family

\section{References}

' Evans H. Chromosome anomalies among live births. $J$ Med Genet 1977:14:309-12.

2 de la Chapelle A. Schroder J. Stenstrand K. et al. Pericentric inversions of human chromosomes 9 and 10. Am J Hum Genet 1974:26:746-66.

${ }^{3}$ Cantu JM, Hernandez A, Vaca G, et al. Trisomy 22q12-qter: 'aneusomie de recombinaisson' of a pericentric inversion. Ann Genet (Paris) 1981;24:37-40.

${ }^{4}$ Fujimoto A, Wilson MG, Towner JW. Duplication of the segment q12.2-qter of chromosome 22 due to paternal inversion 22(p13q12.2). Hum Genet 1983;63:82-4.

5 Funderburk SJ. Sparkes RS. Klisak I. Phenotypic variation in two patients with a ring chromosome 22. Clin Genet 1979:16:305-10.

"Hook EB. Exclusion of chromosomal mosaicism: tables of $90 \%$, $95 \%$ and $99 \%$ confidence limits and comments on use. Am J Hum Genet 1977:29:94-7.

7 Jacobs PA. Frackiewiez A. Law P. Incidence and mutation rates of structural rearrangements of the autosomes in man. $A m J$ Hum Genet 1972:35:301-9.

* Trunca C. Opitz JM. Pericentric inversion of chromosome 14 and the risk of partial duplication of $14 \mathrm{q}$ (14q31-14qter). A $\mathrm{m} \mathrm{J}$ Med Genet 1977:1:217-28.

"Palmer CG. Hodes ME. Reed T. Kojetin J. Four new cases of ring 21 and 22 including familial transmission of ring $21 . J$ Med Genet 1977:14:54-60.

11 Stoll C. Roth MP. Segregation of a 22 ring chromosome in three generations. Hum Genet 1983;63:294-6.

"Hunter AGW. Ray M. Wang HS. Thompson DR. Phenotypic correlations in patients with ring chromosome 22. Clin Genet 1977:12:239-49.

12 Penchaszadeh VB. Coco R. Trisomy 22. Two new cases and delineation of the phenotype. J Med Genet 1975:12:193-9.

$13 \mathrm{Kim}$ HJ. Hsu LYF. Goldsmith LC. Strauss L. Hirschhorn K. Familial translocation with partial trisomy of 13 and 22: evidence that specific regions of chromosomes 13 and 22 are responsible for the phenotype of each trisomy. J Med Genet 1977;14:114-9.

Correspondence and requests for reprints to $\mathrm{Dr}$ Jessie L Watt, Department of Genetics, University of Aberdeen, Medical School Buildings, Foresterhill, Aberdeen AB9 2ZD. 